\title{
Study on the Characteristics of Contrast-Enhanced Ultrasound and Its Utility in Assessing the Microvessel Density in Ovarian Tumors or Tumor-Like Le- sions
}

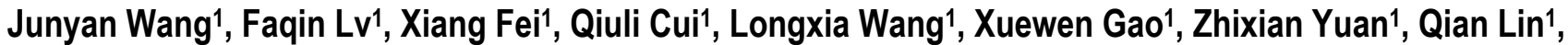 \\ Yali Lv², Aijun Liu ${ }^{凶}$
}

1. Departments of Ultrasound, the Chinese PLA General Hospital, Beijing 100853, China

2. Departments of Pathology, the Chinese PLA General Hospital, Beijing 100853, China

$\triangle$ Corresponding author: Aijun Liu, liuaj@301hospital.com.cn

() Ivyspring International Publisher. This is an open-access article distributed under the terms of the Creative Commons License (http://creativecommons.org/ licenses/by-nc-nd/3.0/). Reproduction is permitted for personal, noncommercial use, provided that the article is in whole, unmodified, and properly cited.

Received: 2010.09.01; Accepted: 2011.05.06; Published: 2011.05.13

\begin{abstract}
Angiogenesis is a critical factor in tumor growth and metastasis, and microvessel density (MVD) was an important parameter for assessing vessels in tumors. However, radiologic assessment of tumor vascularity is not yet well established. In our study, we aimed at investigating the efficacy of contrast-enhanced ultrasonography (CEUS) in exploring the vascularity of the ovarian tumors or tumor-like lesions to assess the relationship between the parameters of the peak intensity (PI) and area under curve (AUC) on CEUS and MVD in ovarian masses. Compared to the contrast-enhanced ultrasound technique, conventional ultrasound shows limitation in differentiating benign and malignant ovarian tumors. The former is promising in improving the sensitivity of detecting small vessels and blood flow in ovarian tumors. Our results showed clear differences in enhancement patterns between benign and malignant ovary tumors or tumor-like lesions. The $\mathrm{PI}$ and $\mathrm{AUC}$ in the malignant tumors were significantly higher than those in the benign tumors or tumor-like lesions $(p=0.00 \mathrm{I}$ and $=0.0 \mathrm{I}$, respectively). The MVD was $43.1 \pm 20.4$ in the benign tumors or tumor-like lesions and was $65.3 \pm 22.3$ in the malignant ones $(p=0.01)$. In both the benign and malignant groups, the PI and AUC were correlated significantly with the MVD $(r=0.595, p=0.001 ; r=0.533, p=0.003$, respectively). The $\mathrm{PI}$ and AUC in CEUS can reflect the MVD in ovarin tumors. The PI and AUC of the ovarian masses in the contrast transvaginal sonography show significant correlation with the angiogenesis and may help in assessing tumor vascularity in ovarian masses.
\end{abstract}

Key words: ovarian tumor; ultrasound; contrast agent; microvessel density

\section{Introduction}

Ovarian cancer is the leading cause of death from gynecological malignancy and the fifth most common cause of cancer-related death in women. Angiogenesis is a critical factor for tumor growth and metastasis in a variety of human tumors, including ovarian cancer [1-4]. Therefore, studying the tumor angiogenesis and microvessel density (MVD) can help to assess the prognosis of cancer patients [5-6]. Currently, imaging techniques such as CT, MRI, and ultrasound have been widely used in preoperative diagnosis of ovarian tumors $[7,8]$. Thereinto, ultrasound is a convenient imaging technique. Owing to the lack of reflection from red blood cells and low signal to noise ratio, conventional transvaginal color 
Doppler ultrasound shows limitation in differentiating ovarian tumors with similar acoustic properties, relatively low and small blood vessels, and slow flow [9-11]. CEUS has been used preoperatively in diagnosing ovarian tumor as well as for correlating ultrasound characteristics and tumor angiogenesis [12-18]. In 1994, Suren [19] reported that Levovist (Schering, Berlin, Germany) succeeded in enhancing the Doppler signal strength in small ovarian tumors. Ten years later, Testa et al. [20] reported the utitility of second-generation ultrasound contrast agent SonoVue (Bracco, Italy) during transvaginal ultrasound for differentiating benign and malignant ovarian masses in 33 patients. In their study [20], CEUS was more accurate in displaying a microvascular bed than conventional Droppler imaging and therefore showed better differentiation ability than the latter.

Studies on angiogenesis of ovarian cancer have demonstrated a significant correlation of the angiogenesis with the prognosis in ovarian cancer [21, 22]. Xie et al [23] found that the peak velocity of ovarian tumor blood flow as detected by CDFI was positively correlated with MVD, reflecting the tumor angiogenesis status. So far, there have been only few reports on transvaginal CEUS characteristics and their relationship with MVD in benign and malignant ovarian lesions. Our study aimed at analyzing the CEUS parameters and their relations to MVD detected by immunohistochemistry in 62 ovarian benign and malignant tumors or tumor-like lesions.

\section{Materials and methods}

\section{Patient selection}

Sixty-two patients (mean age $43.2 \pm 3.9$ years, range 38-74 years) who were diagnosed to have an ovarian mass that was difficult to confirm by conventional ultrasound in our hospital from October 2005 to March 2010 were enrolled in this study. All these patients received a contrast-enhanced transvaginal ultrasound to identify the characteristics and tumor angiogenesis.

\section{Ultrasound examination}

CEUS was performed with an Acuson Sequoia 512 ultrasound system (Siemens Medical Solutions, Mountain View, CA), contrast pulse sequence (CPS), real-time ultrasound imaging software, and EV8-C4 (4-8 MHz) endovaginal probe.

The following procedures were performed: (1) recording of the ovarian mass size, echo characteristics, border, color Doppler flow distribution with conventional transvaginal ultrasound and color Doppler scale; (2) determination of the angiographic tar- get: ultrasound image analysis performed for one ovarian mass side with unclear diagnosis or typical lesions; (3) selection of the plane with solid lesion part, thick cyst wall, a cavity containing papilla, or the most abundant blood supply indicated by CDFI as the best imaging observation view, then switching to contrast pulse sequencing (CPS) with low mechanical index (MI) ranging from 0.12 to 0.16 (the radio frequency signal, $\log$ values and pixel density were used). The probe in all the procedures was maintained in the same position; (4) All patients received injections of a sulfur hexafluoride ultrasound contrast agent (SonoVue; Bracco SpA, Milan, Italy). The agent $(25 \mathrm{mg}$ ) was shaken for about 1 minute with $5 \mathrm{~mL}$ of $0.9 \%$ saline solution, and $4.8 \mathrm{~mL}$ of this suspension was injected as a bolus manually through an 18-gauge cannula placed in the antecubital vein. Then $5 \mathrm{~mL}$ of a $0.9 \%$ saline flush was injected. Seven seconds after the blush count time started, the real-time enhancement pattern of contrast agent inside the tumor was observed for 3-5 minutes and the imaging video was recorded. After scanning, the video was replayed and the area with significant contrast enhancement was chosen as the ROI which was manually outlined. The time-intensity curve (TIC) was drawn automatically with quantitative imaging analysis software (AxiusTM auto tracking contrast quantification, ACQ) to obtain the following parameters under intra-lesion contrast perfusion: arrival time (AT), peak intensity (PI), time to peak intensity (TTP), and the area under the curve (AUC). Data were processed in the same conditions with the same ultrasound system. The enhancement patterns and TIC results were analyzed by two physicians.

\section{Immunohistochemistry}

The ovarian tumors or tumor-like lesions surgically removed were embedded in paraffin, sectioned, and immunohistochemically stained for CD34 with a monoclonal mouse anti-human antibody (Dako Reagent Company, Carpinteria, CA, USA). A positive control (known ovarian cancer section) and a negative control (PBS instead of primary antibody) were used for all stains. Positive CD34 staining was seen in the cytoplasm of vascular endothelial cells. Brown-stained individual vascular endothelial cells or endothelial cell clusters were counted as one microvessel. Vasculature with significant smooth muscle wall and lumen greater than the diameter of eight blood vessels were not counted.

The MVD was determined by the modified quantification method as reported by Weidner et al [24]. Five fields of the most MVD expression particles were observed at low magnification $(\times 100)$, and then 
counted at higher magnification $(\times 200)$. The mean MVD value was calculated.

\section{Statistical analysis}

SPSS 16.0 statistical software was used for data analysis. Quantitative data was expressed as $(\bar{x} \pm s)$. An independent sample t-test was used to compare the differences between the two groups. The ultrasound parameters and MVD in benign and malignant ovarian tumors or tumor-like lesions were compared using bi-variant correlation analysis. $p<0.05$ was considered statistically significant.

\section{Results and Discussion}

\section{The contrast peak intensity, area under the curve in ovarian tumor}

According to the surgical laparoscopic and ultrasound-guided biopsy result, the patients were divided into benign and malignant groups. The benign group $(n=36,58.1 \%)$ included serous papillary cystadenoma $(n=6)$, serous papillary adenofibroma $(n=4)$, endometriosis $(n=5)$, mature teratoma $(n=5$ including 1 struma ovarii), fibrothecomatous tumor $(n=9)$, steroid cell tumor $(n=1)$, ovarian abscess $(n=1)$, and corpus luteum cyst $(n=5)$. The malignant group $(n=26,41.9 \%)$ included serous papillary carcinoma $(\mathrm{n}=14,2$ bilateral), endometrioid adenocarcinoma $(n=2)$, clear cell carcinoma $(n=2)$, fibrosarcoma $(n=1)$, and metastatic carcinoma ( $\mathrm{n}=7,5$ bilateral). The mean diameter of these masses was $4.4 \pm 2.1 \mathrm{~cm}$ (range 1.7 to $9.3 \mathrm{~cm})$, and $38.7 \%(24 / 62)$ of them were less than $3 \mathrm{~cm}$ in diameter, and $50.0 \%(31 / 62)$ less than $5 \mathrm{~cm}$ in diameter.

Our study showed that in benign tumors or tumor-like lesions, CEUS ring-like enhancement was seen in cystic wall and/or papillae of the ovarian cyst and abscess, while solid tumors or tumor-like lesions showed either internal scattered mild enhancement or branching regular moderate low enhancement. In malignant tumors, CEUS showed overall heterogeneous enhancement or dendritic, rapidly increased enhancement. CEUS parameters mainly included PI, AT, TTP and AUC. PI and AUC indicated enhancement intensity, AT and TTP were related to enhancement time. In this study, the CEUS parameters of both benign and malignant ovarian tumors and tumor-like lesions as well as the TIC were shown in Figures (1A-E, 2A-E) and Table 1. PI and AUC were significantly higher in malignant tumors than those in the benign group ( $p=0.001$ and $p=0.01$, respectively), which suggested that malignant ovarian tumors were rich in blood perfusion.
Table I. Comparison of TIC parameters between benign and malignant tumors $(\bar{x} \pm s)$

\begin{tabular}{llllll}
\hline Group & Case & $\mathrm{AT}(\mathrm{s})$ & $\mathrm{TTP}(\mathrm{s})$ & $\mathrm{PI}(\mathrm{dB})$ & $\mathrm{AUC}$ \\
\hline benign & 36 & $16.4 \pm 4.0$ & $27.6 \pm 6.6$ & $12.0 \pm 6.8$ & $8.8 \pm 6.1$ \\
malignant & 26 & $13.3 \pm 4.8$ & $22.2 \pm 6.5$ & $18.3 \pm 5.7$ & $17.1 \pm 6.5$ \\
\hline p-value & & 0.017 & 0.006 & 0.001 & 0.01 \\
\hline
\end{tabular}

\section{MVD and its relation with CEUS parameters in ovarian tumors}

The MVD by immunohistochemical assessment was $43.1 \pm 20.4$ in the benign tumors or tumor-like lesions, significantly lower than $65.3 \pm 22.3$ in the malignant ones $(p=0.002$, Figures $1 \mathrm{~F} \& 2 \mathrm{~F})$. PI and AUC were positively correlated with MVD in both benign and malignant groups $(r=0.595$ and $r=0.533 ; p=0.001$ and $p=0.003$, respectively; Figures 3A-B). The MVD difference between benign and malignant tumors was correlated with vascular endothelial cell activity and angiogenesis-related features inside tumors [25]. We also found that vascular density changes were significantly different in benign and malignant groups. MVD was well correlated with tumor tissue blood perfusion in both groups, suggesting that the increased malignant tumors MVD can increase blood volume.

There were few limitations in our study. First, some benign ovarian tumors or tumor-like lesions with more blood vessels might show the same ultrasound characteristics as the malignant tumors. Second, although microvessels in both benign and malignant lesions were different, their number and distribution might overlap. In our study, one case of ovarian abscess and one corpus luteum cyst also showed a rapid enhancement and similar CEUS parameters as malignant tumors. This may be related to the increase of blood supply from increased inflammatory granulation tissue in the abscess wall, angiogenesis, and higher perfusion due to an increased estrogen level in the ovarian luteal phase. However, in the above cases, the circle enhancement was shown in the cystic wall, which was different from that in malignant tumors. The rapid enhancement was shown only in the cystic wall of the abscess and corpus luteum cyst, not in solid-like incomplete abscess liquefaction and cystic wall pseudopapillae of the corpus luteum cyst. In addition, the papillary enhancement pattern in one case of struma ovarii overlapped with malignant tumors, and one clear cell carcinoma overlapped with benign tumors. More studies are needed to investigate its contrast-enhanced ultrasonic characteristics in these lesions. Nonetheless, 
ovarian cancer is usually accompanied by metastasis in omentum, peritoneum and lymph node et al. which are helpful features for differentiating them from benign lesions. Finally, the contrast-enhanced imaging parameters of benign and malignant ovarian lesions seldom partially overlapped. Therefore, proper diagnosis should be based on the comprehensive clini- cal data analysis, conventional sonographic features and enhanced ultrasound imaging.

In summary, PI and AUC on CEUS can be applied to evaluate vessels in ovarian tumors or tumor-like lesions and can provide noninvasive parameters for clinical evaluation of tumor vascularity.
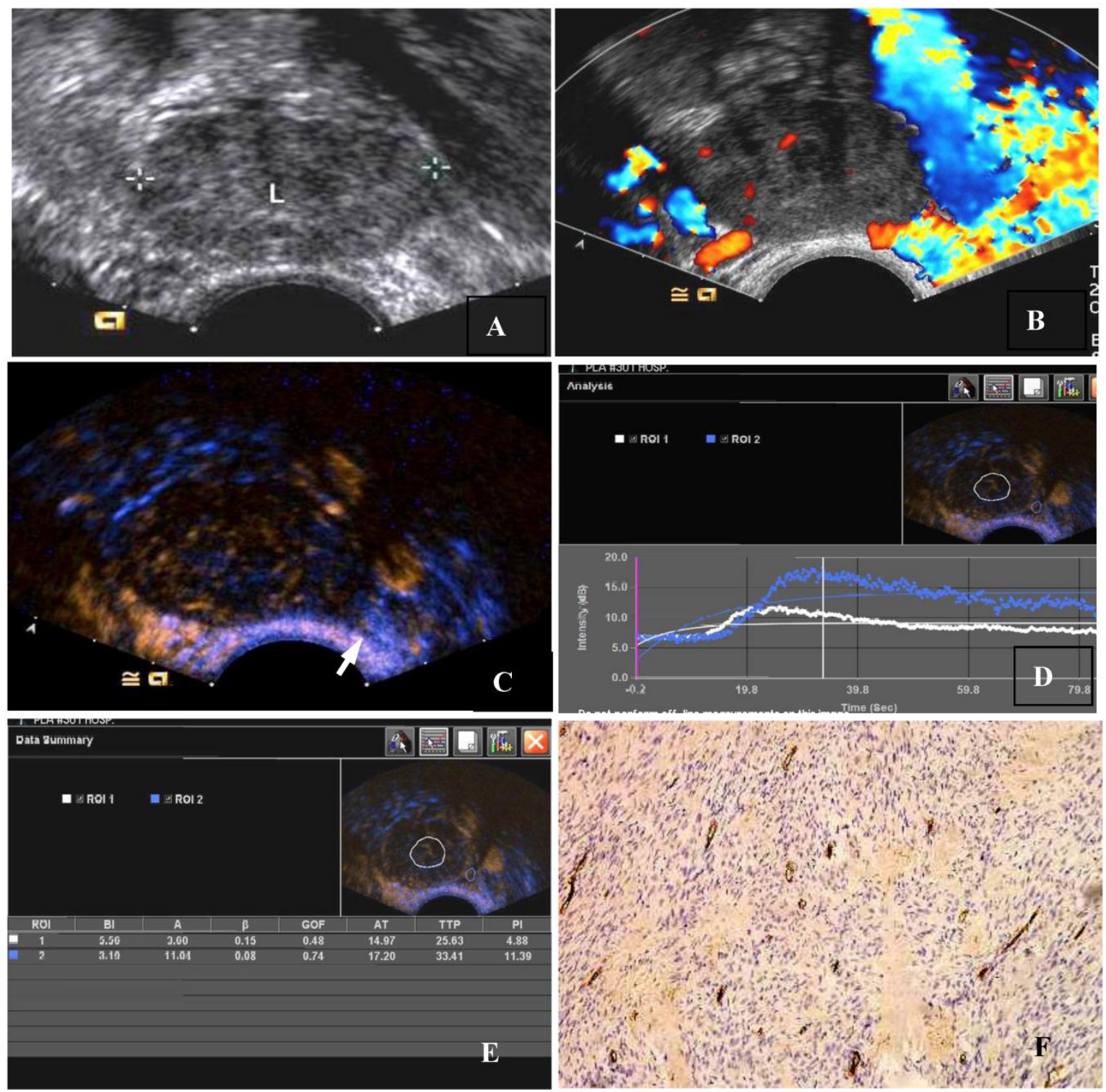

Figure I. The CEUS and MVD of a thecoma of the ovary. (A) Two-dimensional transvaginal ultrasound showed a heterogeneous hypoechoic mass $(3.9 \mathrm{~cm} \times 3.7 \mathrm{~cm} \times 2.7 \mathrm{~cm})$ in the left ovary. (B) Little scattered blood flow signal could be seen in CDFI. (C) CEUS showed slightly scattered enhancement within the ovarian tumor, earlier than surrounding ovarian tissue, the peak intensity of II.88 dB, lower than $18.39 \mathrm{~dB}$ of the surrounding ovarian tissue. (Arrows indicate the mass and the boundaries of the ovary). (D)A region of interest (ROI) was taken from the mass and ovary respectively. (White ROI I: the region of interest within the mass, blue ROI 2: region of interest within the ovarian tissue), drawing TIC (white and blue curves represent the TIC of the mass and ovary). (E)TIC showed AT 21.97 seconds, TTP 32.63 seconds, PI II.88 dB, AUC 10.60, ROI 2 for the ovarian tissue: AT 24.20 seconds, TTP 40.4I seconds, PI 18.39 dB. AUC 18.04. (F) MVD (CD34 immunohistochemical staining, magnification 200x), scarce interstitial microvessels. 

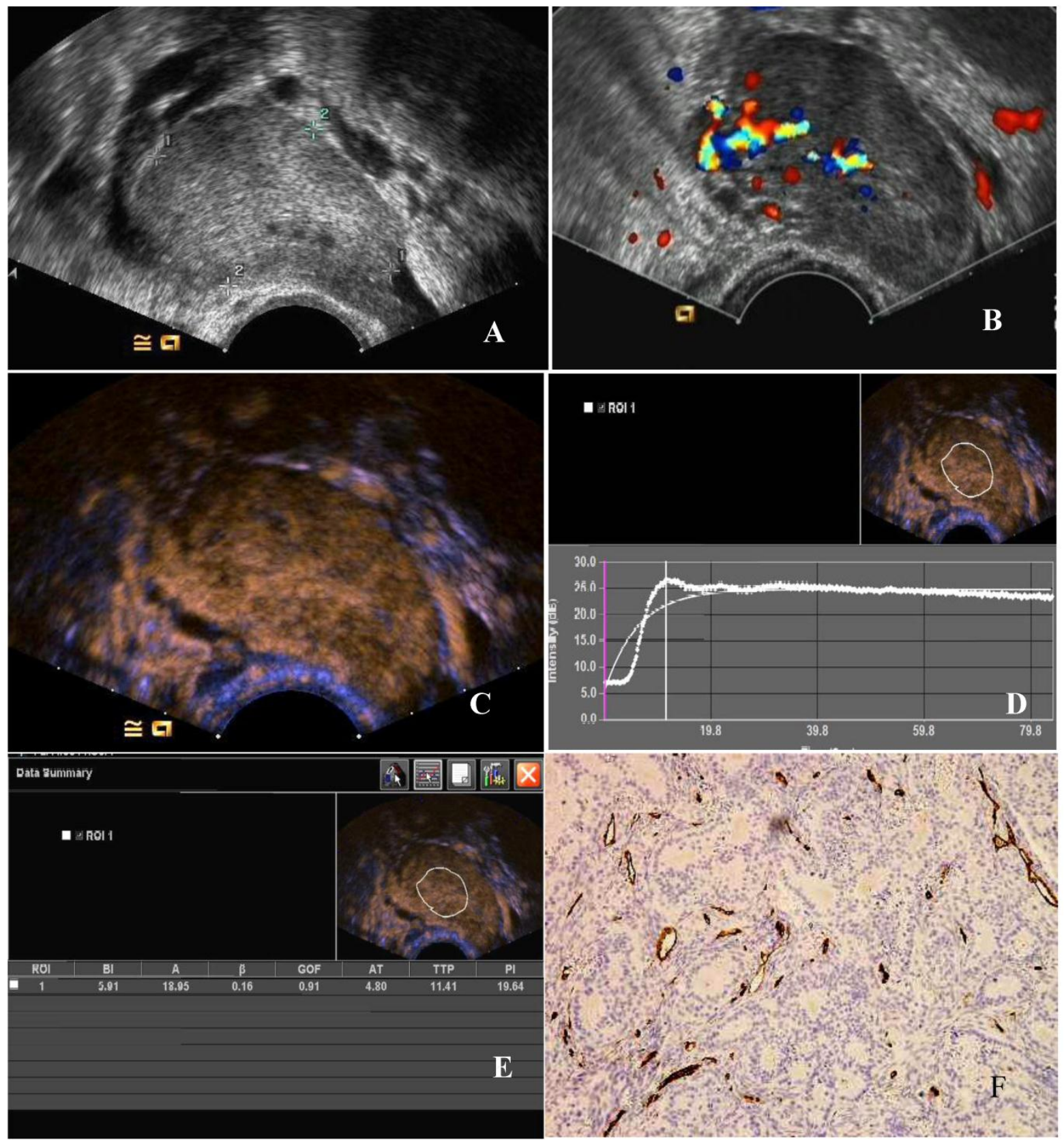

Figure 2. The CEUS and MVD of a poorly -differentiated serous adenocarcinoma of the ovary. (A) Two-dimensional transvaginal ultrasound showed a hypoechoic mass $(4.9 \mathrm{~cm} \times 3.0 \mathrm{~cm} \times 3.3 \mathrm{~cm})$ in the left adnexa with a clear boundary and heterogeneous echo. (B) Abundant blood flow signals can be seen in CDFI. (C) After II.8 seconds of contrast agent injection, a rapid high enhancement can be seen. (D) A region of interest (ROI) within the tumor was taken to draw a TIC (white curve represents the tumor TIC). (E) The tumor TIC showed that AT was II.8 seconds, TTP I8.4I seconds, PI is 26.64 dB, AUC 25.95. (F) MVD showed significantly increased interstitial microvessels (CD34, immunohistochemical staining, magnification 200×). 

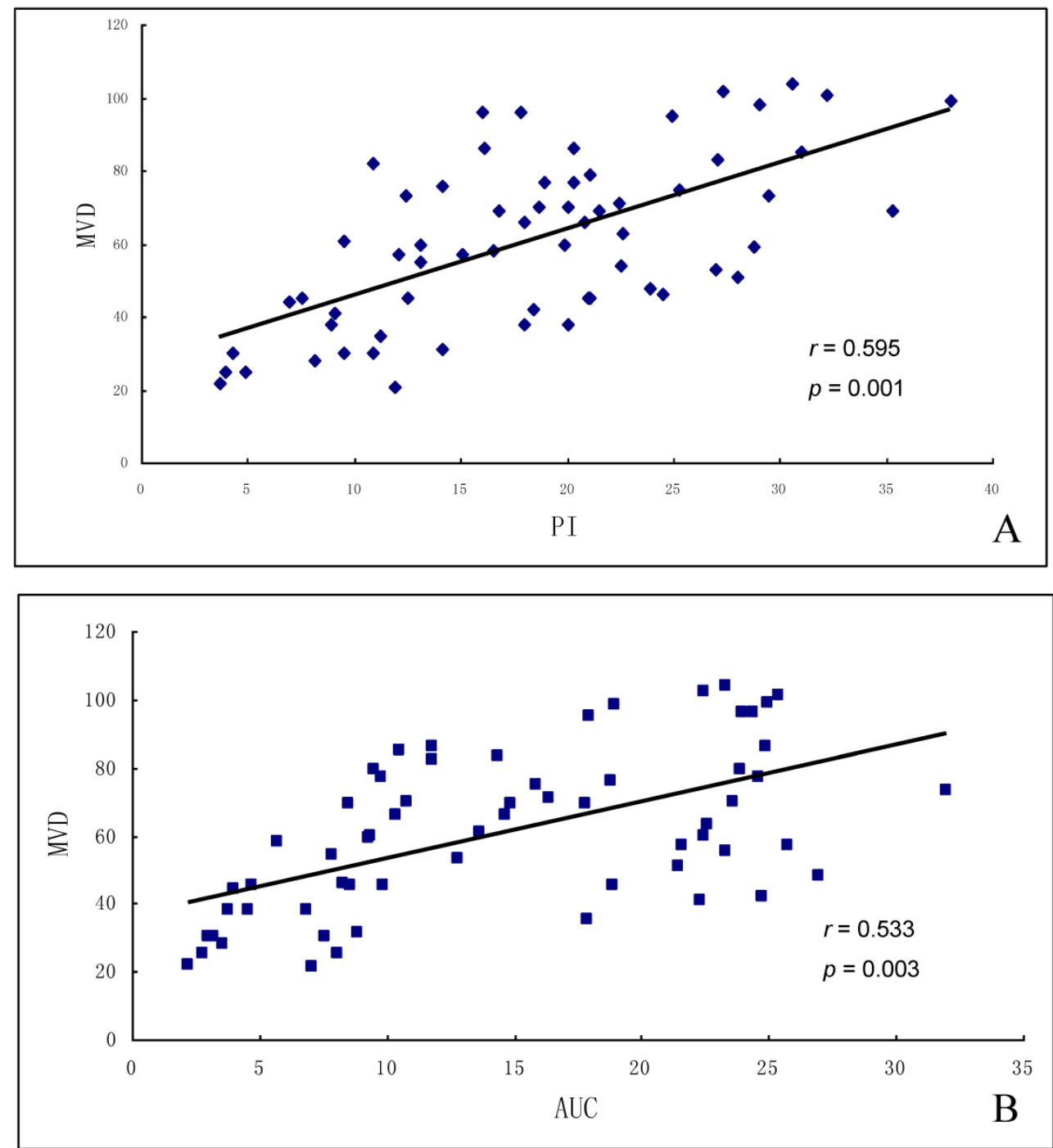

Figures 3. Correlation of MVD and TIC parameters in ovarian tumors. (A) MVD was positively correlated with PI, and (B) MVD was positively correlated with AUC.

\section{Acknowledgement}

This study was supported by Eleventh Five-Year-Plan Military Projects (06MB293) and Military Specialized Projects Fund (06A).

\section{Conflict of Interests}

The authors have declared that no conflict of interest exists.

\section{References}

1 Tang HS, Feng YJ, Yao LQ. Angiogenesis, Vasculogenesis, and Vasculogenic Mimicry in Ovarian Cancer. Int J Gynecol Cancer, 2009, 19 (4): 605-610.

2 Fleischer AC, Niermann KJ, Donnelly EF, et al. Sonographic depiction of microvessel perfusion: principles and potential. J Ultrasound Med, 2004, 23(11):1499-1506.
3 Niermann KJ, Fleischer AC, Huamani J, et al. Measuring tumor perfusion in control and treated murine tumors: correlation of microbubble contrast-enhanced sonography to dynamic contrast-enhanced magnetic resonance imaging and fluorodeoxyglucose positron emission tomography. J Ultrasound Med, 2007, 26(6):749-756.

4 Yankeelov TE, Niermann KJ, Huamani J, et al. Correlation between estimates of tumor perfusion from microbubble contrast-enhanced sonography and dynamic contrast-enhanced magnetic resonance imaging. J Ultrasound Med, 2006, 25(4):487-97.

5 Tan XJ, Lang JH, Lou WZ, et al. prognosis and detection of angiogenesis and MVD in ovary epidermal cancer. Journal of Chinese oncology, 2008, 30(4): 274-278.

6 Sood AK, Fletcher MS, Zahn CM, et al. The clinical significance of tumor cell-lined vasculature in ovarian carcinoma: implications for anti-vasculogenic therapy. Cancer Biol Ther. 2002, 1(6):661-4. 
7 Varras M. Benefits and limitations of ultrasonographic evaluation of uterine adnexal lesions in early detection of ovarian cancer. Clin Exp Obstet Gynecol, 2004, 31(2):85-98.

8 Liu JB, Wang JR. Contrast-enhanced ultrasound imaging. Beijing: Science and Technology Literature publishing house. 2010:3.

9 Valentin L. Use of morphology to characterize and manage common adnexal masses. Best Pract Res Clin Obstet Gynaecol, 2004, 18(1): 71-89.

10 Fleischer AC, Lyshchik A, Jones HW, et al. Contrast-enhanced transvaginal sonography of benign versus malignant ovarian masses: preliminary findings. J Ultrasound Med, 2008, 277 (7): 1011-1018.

11 Wang JY, Cui QL, Wang lx, et al. Study on the application of contrast enhanced transvaginal ultrasound in the differentiation of benign from malignant ovarian leision. Chinese Journal of Medical Ultrasound (electronic edition), 2010, 7 (7): 1152-1161.

12. Mironov S, Akin O, Pandit-Taskar N, et al. Ovarian cancer. Radiol Clin North Am. 2007, 45(1): 149-166.

13 Marret H, Brewer M,Giraudeau B, et al. Assessment of cyclic changes of microvessels in ovine ovaries using sonovue contrast-enhanced ultrasound. Ultrasound in Med. \& Biol, 2006, 32(2):163-169.

14 Orden MR, Jurvelin JS, Kirkinen PP. Kinetics of a US contrast agent in benign and malignant adnexal tumors. Radiology, 2003,226 (2): 405-410.

15 Marret H, Sauget S, Giraudeau B, et al. Contrast-enhanced sonography helps in discrimination of benign from malignant adnexal masses. J Ultrasound Med, 2004, 23 (12): 1629-1639.

16 Dai Q, Liu ZZ, Jiang YX, et al. Transvaginal contrast-enhanced sonography in benign and malignant adnexal masses. Chin J of Ultrasonography. 2006, 15 (9): 693-697.

17 Fleischer AC, Lyshchik A, Lyshchik A, et al. Diagnostic Parameters to Differentiate Benign From Malignant Oarian Masses With Contrast-Enhanced Transvaginal Sonography. J Ultrasound Med, 2009, 28(10):1273-1280.

18 Fleischer AC, Lyshchik A, Andreotti RF, et al. Advances in sonographic detection of ovarian cancer: depiction of tumor neovascularity with microbubbles. AJR, 2010, 194(2): 343-348.

19 Suren A, Osmers R, Kulenkampff D, et al . Visualization of blood flow in small ovarian tumor vessels by transvaginal color Doppler sonography after echo enhancement with injection of Levovist. Gynecol Obstet Invest , 1994,38 (3) :210-212.

20 Testa A C, Ferrandina G, Fruscella E, et al. The use of contrasted transvaginal sonography in the diagnosis of gynecologic diseases:a preliminary study. J Ultrasound Med, 2005, 24(9):1267-1278.

21 Palmer JE, Sant Cassia LJ, Irwin CJ, et al. Prognostic value of measurements of angiogenesis in serous carcinoma of the ovary. Int J Gynecol Pathol, 2007, 26(4):395-403.

22 Labiche A, Elie N, Herlin P, et al. Prognostic significance of tumour vascularisation on survival of patients with advanced ovarian carcinoma. Histol Histopathol. 2009, 24(4):425-435.

23 Xie HN, Li LJ, Kong QY, et al. study on correlation of MVD in ovarian malignant tumor and ultrasonic blood flow index. Chinese Journal of practical gynecology and obstetrics. 2002, 18 (9): 553-554.

24 Weidner N,Semple JP,Welch WR, et al. Tumor Angiogenesis and metastasis-correlation in invasive breast carcinoma. N Engl J Med, 1991, 324(1):1-8.

25 Emoto M, Iwasaki H, Mimura K, et al. Differences in the angiogenesis of benign and malignant ovarian tumors, demonstrated by analysis of color Doppler ultrasound, immunohistochemistry, and microvessle density. Cancer, 1997, 80 (5): 899-907. 